APLHA 99-03

\title{
A New Technique for Sampling Multi-Modal Distributions
}

\author{
K.J. Abraham \\ Department of Physics and Astronomy \\ Iowa State University \\ Ames IA 50011 \\ e-mail abraham@iastate.edu \\ L.M. Haines \\ Department of Statistics and Biometry \\ University of Natal \\ Private Bag X01 \\ 3209 Scottsville \\ Pietermaritzburg \\ South Africa \\ e-mail haines@stat.unp.ac.za
}

\begin{abstract}
In this paper we demonstrate that multi-modal Probability Distribution Functions (PDFs) may be efficiently sampled using an algorithm originally developed for numerical integration by monte-carlo methods. This algorithm can be used to generate an input PDF which can be used as an independence sampler in a Metropolis-Hastings chain to sample otherwise troublesome distributions. Some examples in one, two, and five dimensions are worked out. We also comment on the possible application of our results to event generation in high energy physics simulations.(Subj. Classif. 68U20, 65C05, 81V25, 81V15. Keywords Monte Carlo Optimisation, Metropolis-Hastings Chain, Vegas Algorithm, Independence Sampler).
\end{abstract}


The key to solving a wide range of optimisation problems in science and engineering lies in being able to efficiently sample a (possibly very complex) $\mathrm{PDF}$ in one or more dimensions. In many cases of interest, this requires inverting an integral which may not be possible by analytical or semi-analytical means. In such circumstances, efficient computer algorithms are crucial. The perhaps best known such algorithm is the Metropolis algorithm [1], which can in principle be used to generate an accurate sample from any PDF no matter how complex, by a guided random walk. However, the Metropolis algorithm is potentially inefficent when confronted with a PDF with multiple modes, or peaks, especially if they are well seperated. As is well known, a very large number of random steps may needed to locate a new mode, once one mode has been discovered, leading to a dramatic drop in the efficiency of the scheme. In this paper we will show how this problem can be circumvented in a certain class of problems.

In order to make the subsequent discussion more clear, we will present a brief analysis of the weakness of the Metropolis scheme outlined in the previous paragraph. Let $\vec{X}_{i}$ be some randomly choosen point in the space where the PDF of interest $\Pi$ (not necessarily normalised), is to be sampled. A new point $\vec{X}_{f}$ at a distance $\delta$ from $\vec{X}_{i}$ is choosen and the ratio $\frac{\Pi\left(\vec{X}_{i}\right)}{\Pi\left(\vec{X}_{f}\right)}$ is evaluated. If this ratio is larger than one, then the move $\vec{X}_{i} \rightarrow \vec{X}_{f}$ is accepted. Otherwise it is accepted with probability $\frac{\Pi\left(\vec{X}_{i}\right)}{\Pi\left(\vec{X}_{f}\right)}$. As can be imagined, locating a single peak of $\Pi$ can be easily accomplished. However, moving from one peak to another separated by a distance which is large compared with the stepsize $\delta$ may require a long succession of steps "against the grain"; the net probability of such a sequence is sometimes so small that a prohibitively large number of trials may be needed in order to establish the existence of the second peak. This in a nutshell, is the reason for the potential inefficiency of the Metropolis algorithm alluded to earlier.

One plausible remedy, varying $\delta$ with each move has been incorporated into the Metropolis-Hastings algorithm [2], where the sequence of steps is made on the basis of a proposal distribution. If the proposal distribution mimics $\Pi$, then all the peaks of $\Pi$ may be found without difficulty. However, without prior knowledge of the separation between the peaks of $\Pi$, it is difficult to make a suitable choice for the proposal distribution. In other words, $\Pi$ must be mapped out globally in the region of interest before it has even 
been studied. This requirement may appear to present an insurmountable obstacle to the use of the Metropolis-Hastings algorithm; the rest of this paper deals with methodology we have developed to deal with this problem.

The key to our approach is the observation that the global structure of $\Pi$ is required for another seemingly different problem, the evaluation of the definite integral of $\Pi$ over the region of interest. One technique for doing do which is easily adapted to integrands of higher dimensions is adaptive Monte Carlo simulation. A number of points are thrown at random along the boundaries of the region of interest (defining a grid) and the function is evaluated at these points. This process is repeated, however the second time around the grid from the first iteration is refined so that it is finer in regions where the function is larger and coarser where the function is smaller. On the third iteration, the grid previously obtained is further refined, and so on. After a suitable number of iterations a reliable estimate of the integral may be obtained, for a large class of integrands of interest. Several different variants of this basic algorithm have been developed; we use the VEGAS algorithm [3]. In VEGAS the grid points are used to subdivide the axes into a maximum of fifty bins. The bin boundaries may be used to break up the region of integration into a number of hypercubes. Ideally, the boundaries of the hypercubes are such that $\Pi$ integrated over each hypercube gives the same contribution to the definite integral of $\Pi$ over the region of interest. Smaller hypercubes would then correspond to regions where $\Pi$ is large, larger hypercubes to regions where $\Pi$ is small.

Quite apart from the definite integral, the grid information may also be used to define a PDF $\mathcal{P}$ which roughly mimics $\Pi$. Sampling from $\mathcal{P}$ is straightforward; hypercubes are picked at random in such a way that the probability of picking any given hypercube is the same for all hypercubes, and a random number is used to locate a point $\vec{X}$ in the hypercube by uniform sampling. $\mathcal{P}$ is defined so that it is the same for all points in a given hypercube, and the value of $\mathcal{P}$ in a hypercube of volume $\Delta V$ is $\frac{1}{\Delta V}$. More specifically, in one dimension a random number is used to pick a bin along the $x$ axis in such a way that the probability of picking any bin is the same. Then a second random number is used to pick a point within the bin, all points within the bin sampled uniformly. $\Delta V$ is the bin width, so $\mathcal{P}$ for the point chosen is defined as the inverse of the width of the bin in which the point is located, independent of the precise point choosen in the bin. In two dimensions two random numbers are used to pick an area 
element, and another two random numbers are used to pick a point in the area element. $\Delta V$ is now the area, so $\mathcal{P}$ at the point chosen is defined to be the inverse of the area element. In effect, we have sampled the function globally and have used VEGAS to adaptively construct a PDF $\mathcal{P}$ which is different from $\Pi$ which nonetheless mimics $\Pi$. This procedure can obviously generalised to arbitrarily high dimensions. Regions where $\Pi$ is large (small) corespond the regions where $\Delta V$ is small (large) and hence to regions where $\mathcal{P}$ is large(small).

Our strategy for sampling from $\Pi$ amounts to setting up a MetropolisHastings chain using $\mathcal{P}$ as a proposal distribution. From the discussion in the previous paragraph it is clear that regions where $\Pi$ are large are more likely to be selected than where $\Pi$ is small. A move $\vec{X}_{i} \rightarrow \vec{X}_{f}$ is accepted (rejected) if

$$
\frac{\Pi\left(\vec{X}_{i}\right)}{\mathcal{P}\left(\vec{X}_{i}\right)} \times \frac{\mathcal{P}\left(\vec{X}_{f}\right)}{\Pi\left(\vec{X}_{f}\right)}>r n(<r n)
$$

where $r n$ is a random number uniformly distributed between 0 and 1 . Essentially, we are using $\mathcal{P}$ as an independence sampler for $\Pi$. This method does preserve the condition of detailed balance and the stationary distribution of the resulting Markov Chain does indeed correspond to $\Pi[$ [ 4 ]. Note that the fixed step size $\delta$ plays no role whatsoever, rather $\delta$ varies from move to move tuned to the seperation between the peaks of $\Pi$. One potential objection to this scheme is that the function must be evaluated a large number of times by VEGAS before a random sample can be drawn from it and it is not obvious whether the number of function evaluations needed is less than would be required in an approach with fixed step-size. This objection will be addressed in the example we consider.

The first and simplest example we consider is a mixture of univariate gaussians defined in the interval $[0,22]$. The precise function $\Pi$ is given by

$$
.5\{\mathcal{N}(x, 3,1)\}+.2\{\mathcal{N}(x, 14, .025)\}+.3\{\mathcal{N}(x, 19, .75)\}
$$

where $\mathcal{N}\left(x, \bar{x}, \sigma^{2}\right)$ denotes a uni-variate gaussian with mean $\bar{x}$ and variance $\sigma^{2}$. This function clearly has well-seperated multiple peaks; generating a sample from this PDF of this kind is thus liable to be problematic.

The first step in our approach is to integrate $\Pi$ with VEGAS preserving the grid information generated by VEGAS. In this case the grid information is a set of 50 points in the interval $[0,22]$. The points define bins which are 
such that the contribution to the definite integral from each bin is nearly equal. As expected, the bins are narrow (wide) where the integrand is large (small). П was evaluated 2500 times for this purpose and a grid reflecting the peaks in $\Pi$ was used to generate bins of varying widths. These bins were used to define $\mathcal{P}$ in the interval $[0,22]$ along the lines just described. $\mathcal{P}$ thus obtained has been plotted in Fig. 1; the correspondence between Fig. 1 and Eq. 目 is striking.

The next step is to generate a sample from $\Pi$ using $\mathcal{P}$ as an independence sampler. The acceptance rate of the Metropolis-Hastings chain is remarkably high, about $80 \%$; i.e. about $80 \%$ of the moves were accepted using the criterion defined in Eq. 1. This is desirable from the point of view of minimising CPU time and reflects the accuracy with which $\mathcal{P}$ mimics the underlying distribution $\Pi$ defined in Eq. 2. In all, $\Pi$ was evaluated a total of 15,000 times to generate a sample. We have checked that the average value of the random variable as well as a number of higher moments are correctly reproduced, within statistical error bars. This implies not only that all peaks have been discovered but crucially, that the relative weights of all the peaks have also been correctly reproduced. By way of comparision, we have checked that running a Metropolis chain with the $\Pi$ evaluated over 100000 times with fixed step size does not convincingly reproduce even the first two moments. The advantage of our approach is clear.

We now go on to two dimensional examples. Here a complication arises; in dimensions larger than one the VEGAS algorithm implicitly assumes that $\mathcal{P}$ is factorisable; i.e. $\mathcal{P}$ may be accurately represented in the form $\mathcal{P}=p_{i}\left(x_{i}\right) p_{j}\left(x_{j}\right) \cdots$. For many functions of interest this is a reasonable approximation, however if the function has a peak along a lower dimensional hypersurface other than a co-ordinate axis, this approximation may be a poor one. In particular, the VEGAS algorithm performs poorly if the function (assumed to be defined in a hypercube) has a peak along a diagonal of the hypercube. However, this does not mean that the distribution $\mathcal{P}$ generated from the VEGAS grid cannot be used to sample from $\Pi$. All that happens is that the acceptance rate of the resulting Metropolis chain is lower. To illustrate this point, we consider a mixture of two bi-variate gaussians in a square whose means lie along a diagonal. The precise function is defined below.

$$
\Pi=0.7\{\mathcal{G}(x, y, 4,4,1,1, .8)\}+0.3\{\mathcal{G}(x, y, 12,12,1,1,-.8)\}
$$


where $\mathcal{G}\left(x, y, \mu_{x}, \mu_{y}, \sigma_{x}, \sigma_{y}, \rho\right)$ is defined by

$$
\frac{1}{2 \pi \sigma_{x} \sigma_{y} \sqrt{1-\rho^{2}}} \exp \frac{-1}{2\left(1-\rho^{2}\right)}\left[\frac{\left(x-\mu_{x}\right)^{2}}{\sigma_{x}^{2}}+\frac{\left(y-\mu_{y}\right)^{2}}{\sigma_{y}^{2}}-\frac{2 \rho\left(x-\mu_{x}\right)\left(y-\mu_{y}\right)}{\sigma_{x} \sigma_{y}}\right]
$$

The region of integration is an $(16 \times 16)$ square with one corner at the origin and sides along the positive $x$ and $y$ axes. This function is not well suited to evaluation by VEGAS as both peaks lie along a diagonal of the square, and this is reflected in the fact that the acceptance rate of the Metropolis-Hastings chain is only $\sim 23 \%$. However, the grid information does correctly reflect the location of both peaks and the values of $\left\langle x^{n} y^{m}>\right.$ where $(m+n) \leq 6$ are corectly reproduced, indicating not only that both peaks have been found, but also that the relative weights assigned to both peaks is correct. As a check, we have considered another function,

$$
\Pi=0.7\{\mathcal{G}(x, y, 4,4,1,1, .8)\}+0.3\{\mathcal{G}(x, y, 12,4,1,1,-.8)\}
$$

which differs from the bi-variate gaussian in Eq. 3 in that both peaks now lie along a line parallel to the $x$ axis. Once again, grid information is used to generate a sample from which correct moments can be recovered. This time though, due to the more favourable location of the peaks the acceptance rate is almost twice as high as previously. We see again that an adaptive Monte Carlo approach can generate an independence sampler for a Metropolis-Hastings chain even when the target distribution $\Pi$ is two dimensional and has well seperated modes. It is worth pointing out that modifying $\Pi$ by the introduction of stepping stone distributions [5] has been suggested as a means to facilitate sampling PDFs of this nature; in our approach no such modifications are necessary.

We conclude with a brief discussion of the relevance of our methods for event generation in experimental high energy physics simulations, where a sample from a potentially very complicated differential scattering crosssection dependent on more than two variables is required. If analytic inversion is not possible (as is often the case), another approach such as rejection sampling is needed. This however requires an enveloping distribution which must be somehow obtained, either by guesswork or possibly by using the VEGAS grid information [6]. Alternatively the grid information may be used to construct an importance sampler for a Metropolis-Hastings chain which can be used to generate events. To test this in practise, we have considered 
the example of anomalous single $t$ production in future $\gamma \gamma$ colliders, followed by $t \rightarrow b \ell \nu$ evaluated in the narrow width approximation for the $t$ and $W$ [0]. The five dimensional phase space has been integrated over with VEGAS and the resulting grid was used as an importance sampler to generate events along the lines of the previous examples. Neglecting the effects of cuts, smearing and hadronisation, we obtained an acceptance rate of about $75 \%$, even though no attempt whatsoever was made to optimise the grid. In particular, our sampling did not make any use of simplifications resulting either from the use of the narrow width approximation or from the $(V-A)$ structure of weak decays. This suggests that the methods we have outlined may be worthwhile incorporating into event generators for high energy physics, at least in instances when the phase space can be integrated over with VEGAS. Acknowledgments KJA wishes to thank Prof. Krishna Athreya for valuable encouragement and useful discussions, and Prof.J. Hauptman for reading a preliminary version of the manuscript. 


\section{References}

[1] N. Metropolis et.al.; "Equations of state calculations by a fast computing machine" J.Chem.Phys. 21:1087-1091 (1953).

[2] W.K. Hastings; "Monte Carlo sampling methods using Markov Chains and their applications" Biometrika 57: 97-109 (1970).

[3] P. Lepage; " A New Algorithm for Adaptive Multidimensional Integration" J.Comp.Phys. 27:192 (1978).

[4] L. Tierney; "Markov Chains for Exploring Posterior Distributions" Ann.Statist. 22:1701-1762 (1994).

[5] N. Sheehan \& A. Thomas; "On the irreducibility of a Markov Chain defined on a space of genotype configurations by a sampling scheme" Biometrics 49:163-175 (1993).

[6] S. Kawabata; "A New Monte Carlo Event Generator for High Energy Physics" Comput.Phys.Commun. 41:127 (1986).

[7] K.J. Abraham, K. Whisnant, \& B.-L. Young; "Searching for an Anomalous $\bar{t} q \gamma$ Coupling via Single Top Quark Production at a $\gamma \gamma$ Collider" Phys.Lett.B 419:381-386 (1998). 


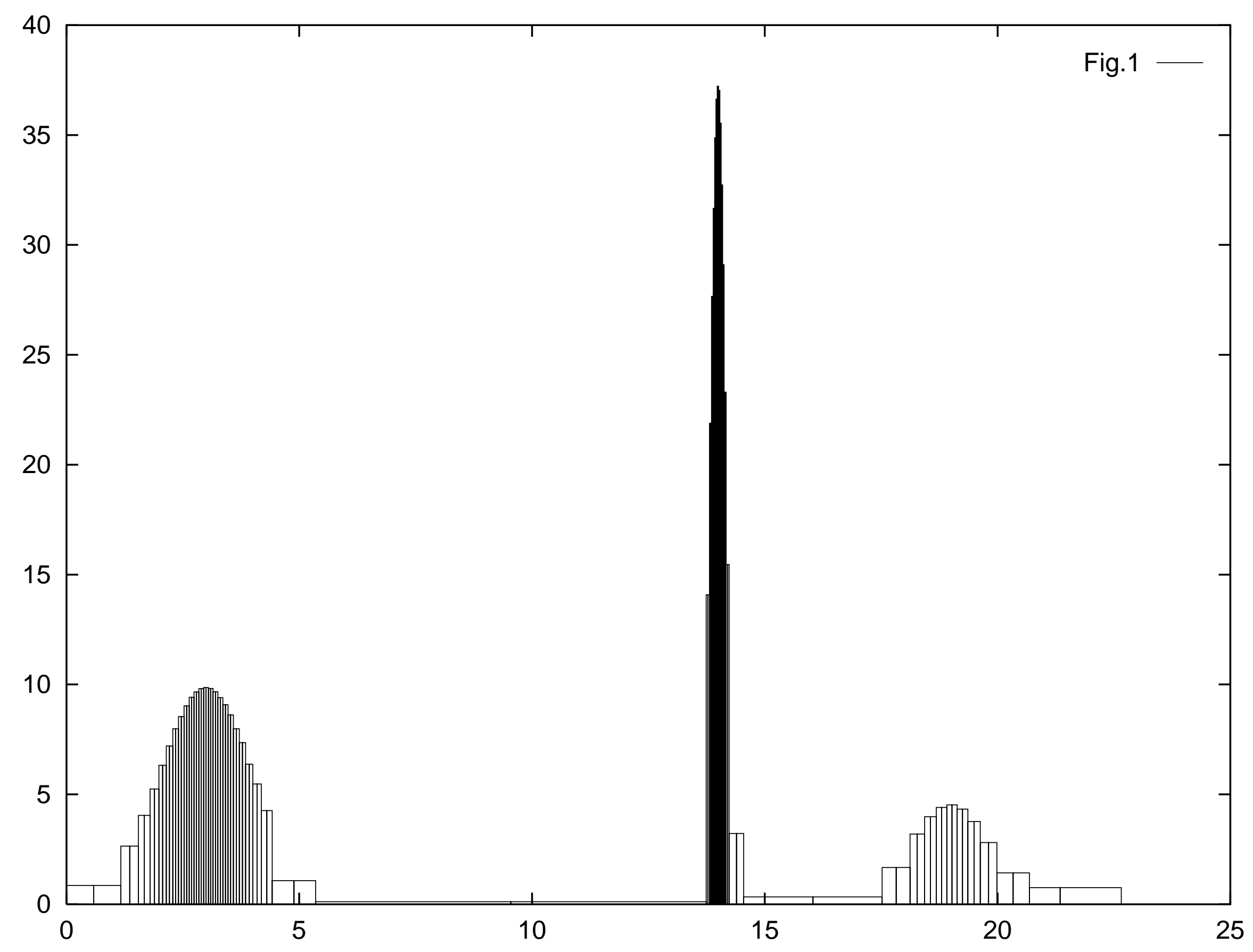

